\title{
Resist Requirements in the Era of Resolution Enhancement Techniques
}

\author{
John S. Petersen and Jeffrey D. Byers
}

\author{
JSP: Petersen Advanced Lithography, Inc., 12325 Hymeadow Drive, Suite 2-201, Austin, TX $78750-1879$ \\ USA, Phone: (512) 241-1100, Fax: (512) 241-1105 \\ jpetersen@advlitho.com
}

JB, 8834 North Capital of Texas Highway, Suite 301, Phone: (512) 381-7369 Fax: (512) 327-1510, Jeff.Byers@kla-tencor.com

\begin{abstract}
At the heart of the tremendous advances of optical microlithography are the resists and the people who drove them to ever-higher performance. In 1980, a resist could reliably image around a $\mathrm{k}_{1}$ of 1.0 to 0.8 . Today without any other extreme RET, resists with anti-reflection coatings production imaging has extended resolution to 0.6 to $0.45 \mathrm{k}_{1}$, effectively doubling the NA of the integrated imaging system. Manipulation of the interrelationships of the physics and chemistry of the imaging process has made this possible. History shows that resists must be designed to best utilize the image being formed in them and that a resist designed for one application may not work for another. This holds true for resolution enhancement techniques as well, for example the quality and brightness of a weak phase-shifted contact depends on the strength of its side lobe, however, if the side lobe is too bright it will print unless the resist is modified not to print it. Also as technology moves to smaller features severe proximity effects make it necessary to adjust resist performance to compensate for these effects. In this discussion we concentrate on the physical chemical effects that makes it possible to extend resolution using resolution enhancement techniques. We will concentrate on energy coupling into the film with high NA imaging at the diffraction limit, the reaction-diffusion reaction and the impact of acid and base diffusion in chemically amplified resists.
\end{abstract}

Keywords: resist, resolution enhancement technique, RET, microlithography, chemically amplified resists, $248 \mathrm{~nm}, 193 \mathrm{~nm}, 157 \mathrm{~nm}$, simulator, antireflection coating, ARC

\section{Introduction}

The cost of designing and taking to market an advanced semiconductor product is becoming prohibitively expensive. Delaying delivery of production quantities of a new chip can cost millions of dollars in lost margins. Having to retool mask sets can cost more than $\$ 1$ million at the $130 \mathrm{~nm}$ technology node and beyond. Lost productivity of a $\$ 2$ billion factory can cost in the neighborhood of $\$ 1$ million per day, and use of these facilities, as laboratories must be reduced to a critical minimum. When considering that it costs each integrated-device-manufacturer (IDM) and foundry $\$ 300$ to $\$ 500$ million to develop a new manufacturing technology-node, and in addition that a state-of-the-art chip like a microprocessor at the 130nm node costs $\$ 18$ million to design and at the $90 \mathrm{~nm}$ node costs around $\$ 50$ million to design with a significant portion being nonrecurring-engineering costs (NRE), it becomes imperative that product is designed so that it not only achieves yield entitlement quickly but does so with the desired performance specifications.

To further complicate things, new yield issues have emerged at sub-180nm process technologies: Performance problems are driven by interconnect wiring efficiency and structure, and gate uniformity and balance. Marginalities give rise to systematic failure where yield loss is no longer driven by random defects but from design and process interactions, of which a significant portion can be traced to litho. Because of this, more conservative design practices are employed to insure greater probability of success; therefore designers are conservatively guard banding because of their lack of understanding of the inherent process.

In reality, beyond the fact that the full power of a process technology isn't being exploited because of excessive guard-banding, there are still manufacturing issues that the current set of EDA tools do not adequately comprehend because of today's increasing process complexity. This means the design community is not fully exploiting the technological capabilities of the IC factory and thus not reaping a higher possible returnon-investment.

Lithography is a large part of this process complexity. If a business is to succeed, understanding image formation and pattern transfer with different types of resolution enhancement techniques and being able to simulate the same becomes imperative for designing and manufacturing integrated circuit products for yield. The industry can no longer afford inaccurate and imprecise design for manufacturing (DFM) practices. 
Ultimately this means we need to use simulation, lots of it, ${ }^{1}$ if we are to succeed. And, excluding the exposure tools for the moment, means that the physical chemistry and material science of resist materials must be well understood and their behavior must be something we can accurately model so that we can find imaging problems or litho related systematic failures a priori, and solve them before they occur in the fab. Secondly as has always been the case the most successful resist products are so because they allow the rest of imaging system to perform at its most cost-effective best.

In this work, we will concentrate on energy coupling into the film with high NA imaging at the diffraction limit, the reaction-diffusion reaction and the impact of acid and base diffusion in chemically amplified resists.

\section{Interference at High Numerical Aperture}

There have been several papers published in the sister conference, Optical Microlithography, dealing with imaging near Brewster's angle ${ }^{2,3}$ and published in Chris A. Mack's book ${ }^{4}$ and the physics will not be discussed here. What it means for the resist and antireflection coating (ARC) optical parameters including thickness is that optimization must take into account the incident angle of the diffracted beams and their polarization. Figure 1 shows for a 100nm chromeless phase-shift lithography 1:3 linewidth-to-space duty cycle the range of the Dill B-parameter that is needed to yield 0.6 microns of depth of focus. As it can be seen, as the resist thickness drops the allowed range of B-values increases as well as a drift upwards. This is because the optimal optical density is usually near $\mathrm{A} /$ micron $=\mathrm{e}^{-15,6,7}$ and in this high numerical aperture and thin resist case varies from around 0.4 to 0.45 . As for the ARC optical parameters it is not enough to find an area that gives good resist profile and dose-to-size, it is important to find an area where the fidelity of the latent image is maintained through focus and exposure. For this type of optimization, using the responses focus-exposure process window size and dose-to-size are the best parameters for study.

\section{Imaging Method Specific Resists}

Historically, resists have been designed with binary masks. To a degree this is okay with weak phaseshift masks because like binary masks they have a zero-order diffraction pattern and the isofocal region is related to the magnitude of that order. ${ }^{8}$ However, as Figure 2 shows the amplitude of the zero-order diffraction pattern is related to the space-to-pitch ratio and the attenuation of the mask material. This is the basis of chromeless phase lithography (CPL). ${ }^{9}$ So designing a resist to have optimal performance with one type of mask and feature may not apply to another. For instance, so called isolated line resists have a large imaging bias that moves the best focus latitude into the target region of the isolated feature it is designed to image. While this resist works fine in this instance it will not work for dense line patterns because their best focal region on the set defocused aerial images is near the target size to begin with and the large imaging bias would shift the best focus-exposure process out of the desired linewidth region. Nor will it work when imaging dense and isolated lines using alternating PSM, because at the optimal sigma for imaging a dense line of normalized pitch of 0.54 , the isolated line's isofocal region is smaller than the target size (and may be at zero linewidth). In this case, as it is for the sidewall-chrome-alternating-aperture (SCAA) mask ${ }^{10,11}$ dense line shown in figure 2, because of balanced opposing electric fields there is no zero-order and the isofocal region is related to the line/pitch ratio and sigma. Thus, to image an isolated line pattern in an open field will take a resist that has a negative imaging bias so as to grow the isofocal region into the target region (with a little mask biasing help) and this leads to our next topic.

\section{OPC with Base Diffusivity (and other resist characteristics)}

Typically, when we think of chemically amplified resists we think of acid diffusion smearing the image and under extreme post-exposure-bake conditions degrading the process window of some or all the features. ${ }^{12}$ As Ted Fedynyshyn is fond of saying, "(In Lithography) All acids diffuse and acid diffusion is bad." Well, Ted is generally right for positive-acting resists. With negatives however, this could be a good thing as we showed in our work with SNR248 ${ }^{\mathrm{TM}}$ from Shipley. ${ }^{13}$ While acid diffusion is generally bad in positive resists, base diffusion can be a very good thing. Especially in the case of the aforementioned alternating isolated lines, where the isofocal region is below the target size. Figure 3 shows with simulation using PROLITH ${ }^{\mathrm{TM}} 3 \mathrm{D}$ v7.2.2 (KLA-Tencor/FINLE Div.), how the isofocal region ${ }^{14,15}$ shifts with base diffusivity for $90 \mathrm{~nm}$ lines for pitches of $180 \mathrm{~nm}$ and $540 \mathrm{~nm}$. In these simulations it is observed that the isofocal region for the $540 \mathrm{~nm}$ pitch moves from $72 \mathrm{~nm}$ to $84 \mathrm{~nm}$, while it moves $8 \mathrm{~nm}$, from $86 \mathrm{~nm}$ to $94 \mathrm{~nm}$ for the $180 \mathrm{~nm}$ pitch with most of its shift occurring once the base's diffusivity exceeds that of the resist's acid. Further note that the depth of focus improved with diffusivity for both pitches but most significantly with the isolated feature. 
This is not the only example of using the resist for OPC. Designing resists so as they do not print subresolution assist features of line features or side-lobes of attenuated phase-shift mask contact hole patterns will also improve image capability. For example, the quality and brightness of a weak phase-shifted contact depends on the strength of its side lobe, however, if the side lobe is too bright it will print unless the resist is modified not to print it. ${ }^{16}$

\section{Multiple-Exposures}

What's next? We are rapidly approaching the limits of single exposures and have begun to use multiple-mask processes to extend resolution to a normalized pitch of 0.54 , but how do we go beyond that? There has been work by people like Steve Brueck ${ }^{17}$ at the University of New Mexico to extend resolution to normalized pitch to 0.5 and below using multiple-exposures with interference lithography and pupil filters. One idea is that one exposure is made processed through transfer and then the process is repeated again until the set of desired features are made. Except for all the processing this is a great idea. We need materials that would make this possible.

To form an image in chemically amplified resists, there is a mundane multiple-exposure process that is more vital to everything that we try to do in advanced imaging that is the exposure first to light and then to heat. We have shown that the post-exposure bake process is not always a simple integrated process ${ }^{18}$ and that the whole thermal cycle is important. ${ }^{19} \mathrm{~A}$ lot of attention is spent on the scanner or stepper but not enough on the hot plate's complete thermal cycle. Figure 4 shows a set of typical thermal cycles for a hot plate. There is a temperature rise time, a set time and a transition time to a cold plate; each has a temperature and time error associated with it. Figure 5 shows a sensitivity function for linewidth change during the post-exposure-bake that was used to generate values in the next figure. Figure 6 shows two tables of ProLE ${ }^{1}$ simulated results with variations in rise time error for a $65 \mathrm{~nm}$ line on a $260 \mathrm{~nm}$ pitch (alternating phase-shift mask) imaged using a low-activation energy resist. The errors are a bit generous but typical of what is observed with this process. The results in the table show that for a budget of $2 \%$ linewidth variation, the post-exposure-bake-temperature control must be better than $\pm 0.25 \mathrm{C}$, and for $1 \%$, it must be better than $\pm 0.07 \mathrm{C}$. This is an area that will need to be further developed by the resist process equipment and the resist manufacturers. Especially at $157 \mathrm{~nm}$, where due to absorbance, making good low activation resists may be problematic and the linewidth sensitivity could be two to three times larger than shown in the figure 6 tables. ${ }^{20}$ Only time will tell.

\section{Conclusion}

Various topics about advanced imaging were covered. Optical parameters for resists and ARC materials need to be optimized for each resist thickness and high numerical aperture and illumination condition. Full vector simulation is required to understand interference correctly in the resist. Different kinds of mask technologies have different kinds of demands on the resist. The desired chemistry of a chemically amplified resist depends on the mask and exposure technology. Isolated line resists for binary masks having totally different requirements than resists for isolated line patterns formed with alternating phase-shift-masks. OPC with resist is critical for driving resolution. Multiple exposures could be a good thing for the industry and as with any imaging technique, materials need to be developed to best exploit its potential. Resists must be insensitive to hot plate variation and hot plates need continued improvement.

Due to time constraints related to publishing this work, line-edge-roughness, pattern collapse, pattern transfer, defect-levels, new electron-beam resists, and immersion lithography are not discussed but are also critical for the success of advanced imaging. But we want to close with this: The most important thing, "Mr./Ms. Resist-maker" is that we can do most anything to extend optical resolution, but the cost of being in business necessitates that the lithographer have access to accurate resist models and parameters to use with them. Properly designed materials are outstanding and almost beyond belief in the resolution they have bought the industry through the years and we thank you (and pay you) for them. But, if we now can't simulate how they (the resists) work so that we can use that knowledge to make better chip designs and imaging processes; then we lose money because we can't ramp to yield fast enough and because we will have more remakes of expensive prototype masks, and many businesses, including yours, will be in jeopardy. Please help us succeed by developing accurate models and good parameters to use with them. Thank you.

\section{Acknowledgements}

The authors would like to thank Dave Gerold and Mark Maslow their support in this work. Also, we would like to thank Chris A. Mack, Mark Smith and Ed Charrier. 
Figures

\section{DoF Dependency on Resist Thickness and Absorbance (Vector Simulation)}

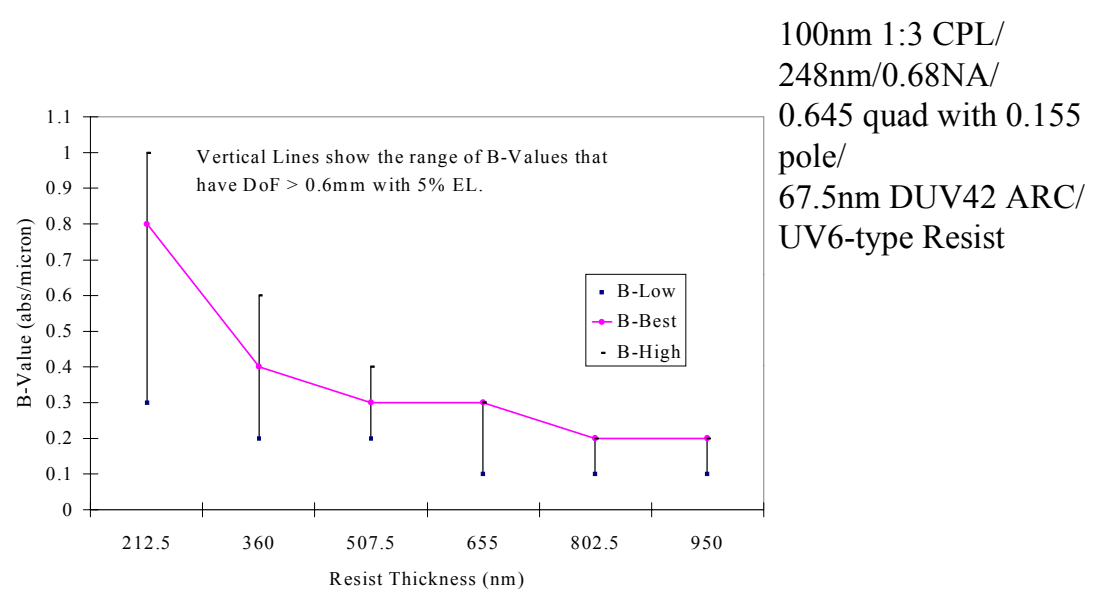

Figure 1: Depth of Focus dependency on resist thickness and absorbance (Vector Simulation) for CPL.

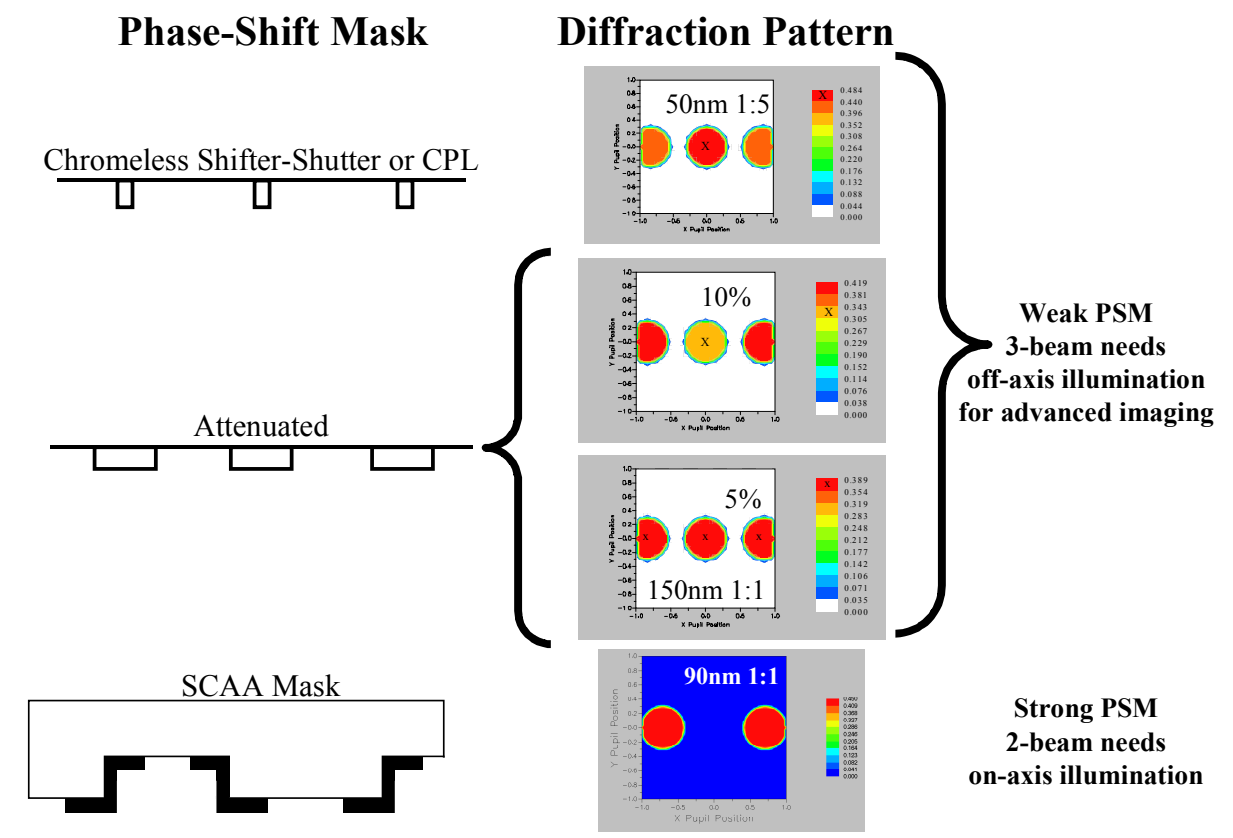

Figure 2: Examples of advanced phase-shift masks for deep subwavelength imaging. CPL masks has $100 \%$, and the attenuated masks have $5 \%$ and $10 \%$ transmission. Weak phase-shifters have a zerodiffraction order, strong phase-shifters do not. 


\section{Isofocal Region Dependence on Base Diffusivity}

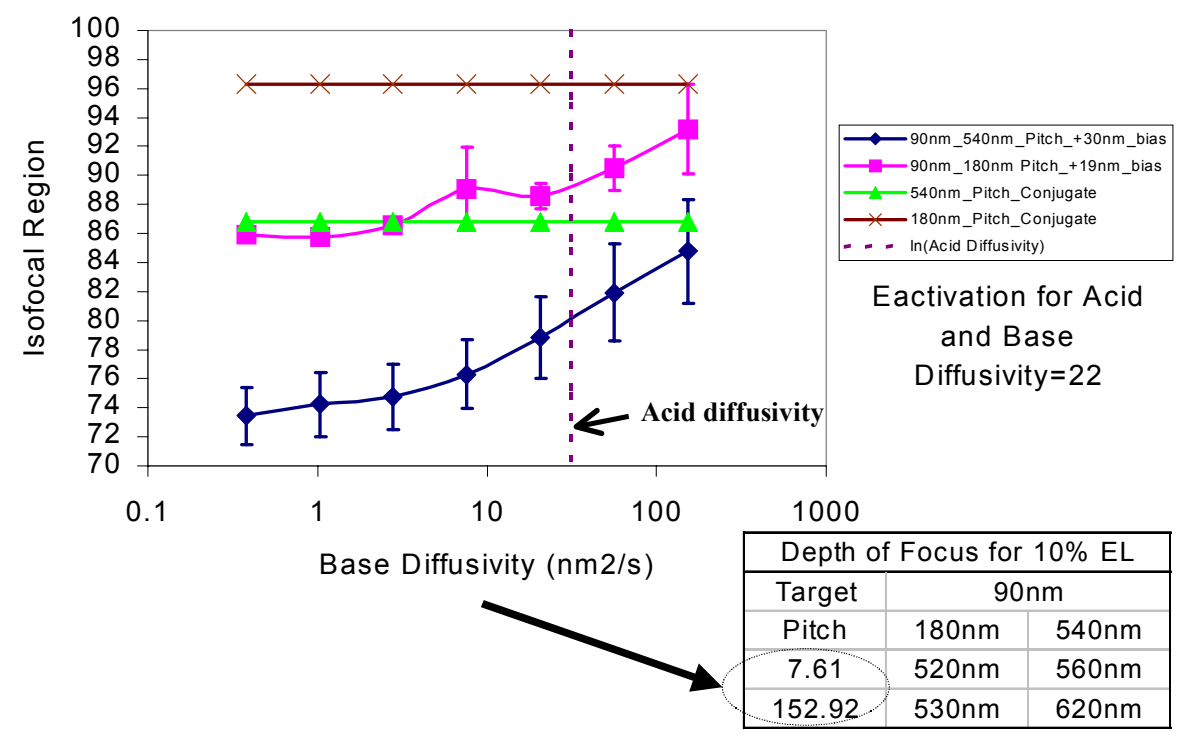

Figure 3: Simulation of isofocal region dependence on base diffusivity for $90 \mathrm{~nm}$ lines with pitches of $180 \mathrm{~nm}$ and $540 \mathrm{~nm}$. Imaged using $193 \mathrm{~nm}$ and 0.75 numerical aperture.

\section{PEB Thermal Profile Stability}

Thermal History Samples

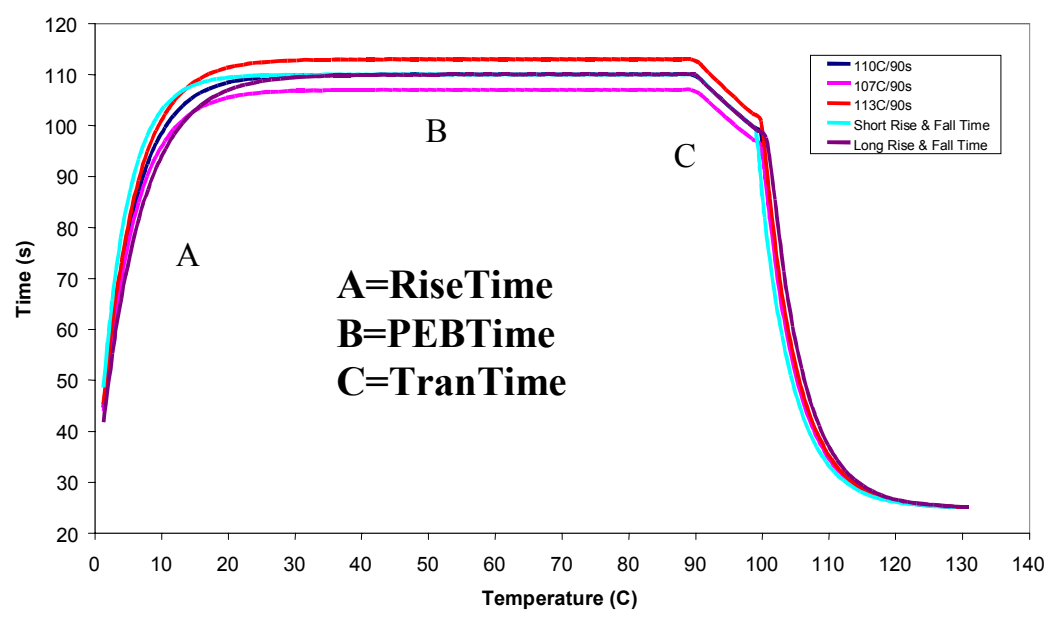

Figure 4: PEB thermal profile stability and definitions. (How will this impact linewidths?) 


\section{Hot Plate Thermal Cycle 1:3 65nm Line Feature Accumulated Error}

$$
\begin{aligned}
\Delta \mathrm{CD}^{2}=\left(\frac{\partial \mathrm{CD}}{\partial \mathrm{Rs}}\right)^{2}(\Delta \mathrm{Rs})^{2}+\left(\frac{\partial \mathrm{CD}}{\partial \mathrm{PC}}\right)^{2}(\Delta \mathrm{PC})^{2}+\left(\frac{\partial \mathrm{CD}}{\partial \mathrm{TC}}\right)^{2}(\Delta \mathrm{TC})^{2}+\left(\frac{\partial \mathrm{CD}}{\partial \mathrm{Ps}}\right)^{2}(\Delta \mathrm{Ps})^{2}+\left(\frac{\partial \mathrm{CD}}{\partial \mathrm{Ts}}\right)^{2}(\Delta \mathrm{Ts})^{2} \\
+\left(\frac{\partial \mathrm{CD}}{\partial \mathrm{Rs}}\right)\left(\frac{\partial \mathrm{CD}}{\partial \mathrm{PC}}\right)(\Delta \mathrm{Rs})(\Delta \mathrm{PC})+\left(\frac{\partial \mathrm{CD}}{\partial \mathrm{Rs}}\right)\left(\frac{\partial \mathrm{CD}}{\partial \mathrm{TC}}\right)(\Delta \mathrm{Rs})(\Delta \mathrm{TC})+\left(\frac{\partial \mathrm{CD}}{\partial \mathrm{RS}}\right)\left(\frac{\partial \mathrm{CD}}{\partial \mathrm{Ps}}\right)(\Delta \mathrm{RS})(\Delta \mathrm{Ps}) \\
+\left(\frac{\partial \mathrm{CD}}{\partial \mathrm{RS}}\right)\left(\frac{\partial \mathrm{CD}}{\partial \mathrm{Ts}}\right)(\Delta \mathrm{RS})(\Delta \mathrm{Ts})+\left(\frac{\partial \mathrm{CD}}{\partial \mathrm{PC}}\right)\left(\frac{\partial \mathrm{CD}}{\partial \mathrm{TC}}\right)(\Delta \mathrm{PC})(\Delta \mathrm{TC})+\left(\frac{\partial \mathrm{CD}}{\partial \mathrm{PC}}\right)\left(\frac{\partial \mathrm{CD}}{\partial \mathrm{Ps}}\right)(\Delta \mathrm{PC})(\Delta \mathrm{Ps}) \\
+\left(\frac{\partial \mathrm{CD}}{\partial \mathrm{PC}}\right)\left(\frac{\partial \mathrm{CD}}{\partial \mathrm{Ts}}\right)(\Delta \mathrm{PC})(\Delta \mathrm{Ts})+\left(\frac{\partial \mathrm{CD}}{\partial \mathrm{TC}}\right)\left(\frac{\partial \mathrm{CD}}{\partial \mathrm{Ps}}\right)(\Delta \mathrm{TC})(\Delta \mathrm{Ps})+\left(\frac{\partial \mathrm{CD}}{\partial \mathrm{TC}}\right)\left(\frac{\partial \mathrm{CD}}{\partial \mathrm{Ts}}\right)(\Delta \mathrm{TC})(\Delta \mathrm{Ts})
\end{aligned}
$$

where Rs = RiseTime; PC = PEBTemp; TC = TransitionTemp; Ps = PEBTime; Ts = Transition time

Figure 5: Hot plate thermal cycle accumulated linewidth error function.

\begin{tabular}{|c|c|r|c|c|c|}
\hline IRSS $\Delta$ CDI & $\begin{array}{c}\Delta \text { Rise } \\
\text { Time }\end{array}$ & $\begin{array}{c}\Delta \text { PEB } \\
\text { Temp }\end{array}$ & $\begin{array}{c}\Delta \text { Trans } \\
\text { Temp }\end{array}$ & $\begin{array}{c}\Delta \text { PEB } \\
\text { Time }\end{array}$ & $\begin{array}{c}\Delta \text { Trans } \\
\text { Time }\end{array}$ \\
\hline $\mathrm{nm}$ & \multicolumn{1}{c|}{$\mathrm{s}$} & \multicolumn{1}{c|}{$\mathrm{C}$} & $\mathrm{C}$ & $\mathrm{s}$ & $\mathrm{s}$ \\
\hline 0.5 & 0.15 & 0 & 2 & 0.1 & 2 \\
\hline 0.6 & 0.15 & 0.0675 & 2 & 0.1 & 2 \\
\hline 0.8 & 0.15 & 0.125 & 2 & 0.1 & 2 \\
\hline 1.1 & 0.15 & 0.25 & 2 & 0.1 & 2 \\
\hline 1.8 & 0.15 & 0.5 & 2 & 0.1 & 2 \\
\hline 3.3 & 0.15 & 1 & 2 & 0.1 & 2 \\
\hline 4.7 & 0.15 & 1.5 & 2 & 0.1 & 2 \\
\hline & & & & & \\
\hline
\end{tabular}

\section{PEB Sensitivity Results for $65 \mathrm{~nm}$}

\begin{tabular}{|c|c|r|c|c|c|}
\hline |RSS $\Delta$ CD & $\begin{array}{c}\Delta \text { Rise } \\
\text { Time }\end{array}$ & $\begin{array}{c}\Delta \text { PEB } \\
\text { Temp }\end{array}$ & $\begin{array}{c}\Delta \text { Trans } \\
\text { Temp }\end{array}$ & $\begin{array}{c}\Delta \text { PEB } \\
\text { Time }\end{array}$ & $\begin{array}{c}\Delta \text { Trans } \\
\text { Time }\end{array}$ \\
\hline $\mathrm{nm}$ & \multicolumn{1}{|c|}{$\mathrm{s}$} & \multicolumn{1}{|c|}{$\mathrm{C}$} & $\mathrm{C}$ & $\mathrm{s}$ & $\mathrm{s}$ \\
\hline 0.7 & 0.3 & 0 & 2 & 0.1 & 2 \\
\hline 0.8 & 0.3 & 0.0675 & 2 & 0.1 & 2 \\
\hline 0.9 & 0.3 & 0.125 & 2 & 0.1 & 2 \\
\hline 1.2 & 0.3 & 0.25 & 2 & 0.1 & 2 \\
\hline 1.9 & 0.3 & 0.5 & 2 & 0.1 & 2 \\
\hline 3.4 & 0.3 & 1 & 2 & 0.1 & 2 \\
\hline 4.8 & 0.3 & 1.5 & 2 & 0.1 & 2 \\
\hline
\end{tabular}

\section{ProLE Simulation} conditions:

Low $\mathbf{E}_{\text {act }}$ Resist 65nm 1:3; 0.75 NA/193nm; alternating PSM

Figure 6: Accumulated linewidth error garnered from simulation results of hot plate thermal cycle study of $1: 365 \mathrm{~nm}$ line:space features imaged using alternating phase-shift masks. 


\section{Optics forever! References}

${ }^{1}$ Petersen, J. S., Maslow, Mark John, and Gerold, Dave J., "Programmable Lithography Engine, ProLETM, Grid-Type Supercomputer and Its Applications", SPIE Vol. 5040, Paper 157 (to be published) (2003).

${ }^{2}$ Brunner, Timothy A., Seong, Nakgeuon, Hinsberg, William D., Hoffnagle, John A. Houle, Frances A., Sanchez, Martha I., "High NA lithographic imagery at Brewster's angle", Proc. SPIE Vol. 4691, Optical Microlithography XV, Anthony Yen; Ed., p. 1-10 (7/2002).

${ }^{3}$ Smith, Bruce W. and Cashmore, Julian, "Challenges in high NA, polarization, and photoresists", Proc. SPIE Vol. 4691, Optical Microlithography XV, Anthony Yen, Ed., p. 11-24 (7/2002).

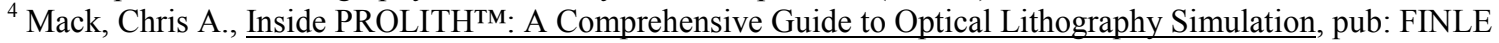
Technologies, Austin, TX, USA, p. 34-45, (1997). (Available from Petersen Advanced Lithography)

${ }^{5}$ Thommes, G., Weber, V., J. Imag. Sci. 29, p. 112 (1985).

${ }^{6}$ Ohfuji, T., Nalamasu, O., Stone, D., J. Vac. Sci. Technol. B11, p. 2714 (1993).

${ }^{7}$ Conley, Willard, Akkapeddi, Ravindra, Fahey, James, Hefferon, George, Holmes, Steve, Spinillo, Gary, Sturtevant, John, Welsh, Kevin, "Improved reflectivity control of APEX-E positive tone deep UV photoresist", SPIE Vol. 2195, Omkaram Nalamasu, Ed., p. $461-477$ (1994).

${ }^{8}$ Smith, Bruce W. and Ewbank, Dale E. “OPC and image optimization using localized frequency analysis", SPIE Vol. 4691, Optical Microlithography XV, Anthony Yen, Ed:, p. 148-157 (2002).

${ }^{9}$ Petersen, John S., Conley, Will, Roman, Bernard J., Litt, Lloyd C., Lucas, Kevin, Wu, Wei, Van Den Broeke, Douglas J., Chen, J. Fung, Laidig, Thomas L., Wampler, Kurt E., Gerold, David J., Socha, Robert J., van Praagh, Judith, Droste, Richard, "Development of a sub-100nm integrated imaging system using chromeless phase-shifting imaging with very high NA KrF exposure and off-axis illumination", Proc. SPIE Vol. 4692, Design, Process Integration, and Characterization for Microelectronics, Alexander Starikov; Kenneth W. Tobin; Eds., p. 298-311 (7/2002).

${ }^{10}$ Gerold, David J., Petersen, John S., Levenson, Marc D., "Multiple Pitch Transmission and Phase Analysis of Six Types of strong phase-shifting masks", SPIE Vol. 4346, Optical Microlithography XIV, Christopher J. Progler, Ed. P. 729-743 (2001).

${ }^{11}$ Petersen, John S., Beach, James V., Gerold, David J., Maslow, Mark J., "Developing an integrated imaging system for the 70-nm node using high numerical aperture ArF lithography", SPIE Vol. 4692, Design, Process Integration, and Characterization for Microelectronics, Alexander Starikov; Kenneth W. Tobin; Eds. , p. 274-287 (2002)

${ }^{12}$ Petersen, John S. and Byers Jeffrey D., "Examination of isolated and grouped feature bias in positive acting, chemically amplified resist systems", SPIE Vol. 2724, Advances in Resist Technology and Processing XIII, Roderick R. Kunz, Ed., p. 163-171 (1996).

${ }^{13}$ Petersen, John S. and Lee, Wei, "Submicron imaging at 248.3nm: A lithographic performance review of an advanced negative resist", SPIE Vol. 1262, Advances in Resist Technology and Processing VII, Michael P. C. Watts, Ed., p. 358-367 (1990).

${ }^{14}$ Petersen, John S. and Kozlowski, "Optical performance and process characterization of several high contrast metalion-free developer processes", SPIE Vol. 633, Optical Microlithography V, Harry L. Stover, Ed., p. 46-56 (1984).

${ }^{15}$ Petersen, John S., "An experimental determination of the optical lithographic requirements for sub-micron projection printing", SPIE Vol. 1088, Optical/Laser Microlithography II, Burn J. Lin, Ed. p. 540-567 (1989).

${ }^{16}$ Petersen, John S., McCallum, Martin, Kachwala, Nishrin, Socha, Robert J., Chen, J. Fung, Laidig, Tom, Smith, Bruce W., "Assessment of a hypothetical roadmap that extends optical lithography through the 70nm technology node", SPIE Vol. 3546, 18th Annual Symposium on Photomask Technology and Management, Brian J. Grenon and Frank E. Abboud, Eds., p. 288-303 (1998).

${ }^{17}$ Zaidi, Saleem H., Brueck, Steven R., "Nonlinear processes to extend interferometric lithography", SPIE Vol. 3676, Emerging Lithographic Technologies III, Yuli Vladimirsky; Ed., p. 371-378 (1999).

${ }^{18}$ Petersen, John S., Byers, Jeffrey D., Carpio, Ronald A., "The formation of acid diffusion wells in acid catalyzed photoresists", Microelectronic Engineering 35, p. 169-174 (1997).

${ }^{19}$ Smith, Mark D., Mack, Chris A., Petersen, John S., "Modeling the impact of thermal history during post-exposure bake on the lithographic performance of chemically amplified resists", SPIE Vol. 4345, Advances in Resist Technology and Processing XVIII, Francis M. Houlihan, Ed., p. 1013-1021 (2001).

${ }^{20}$ Petersen, John S., "Making 157 Work", SEMI Newsletter Service: Lithography Review: Important Developments in Microlithography Vol. 2, Issue 6, Griff Resor, Ed., p. 6-11 (January and February, 2000). 